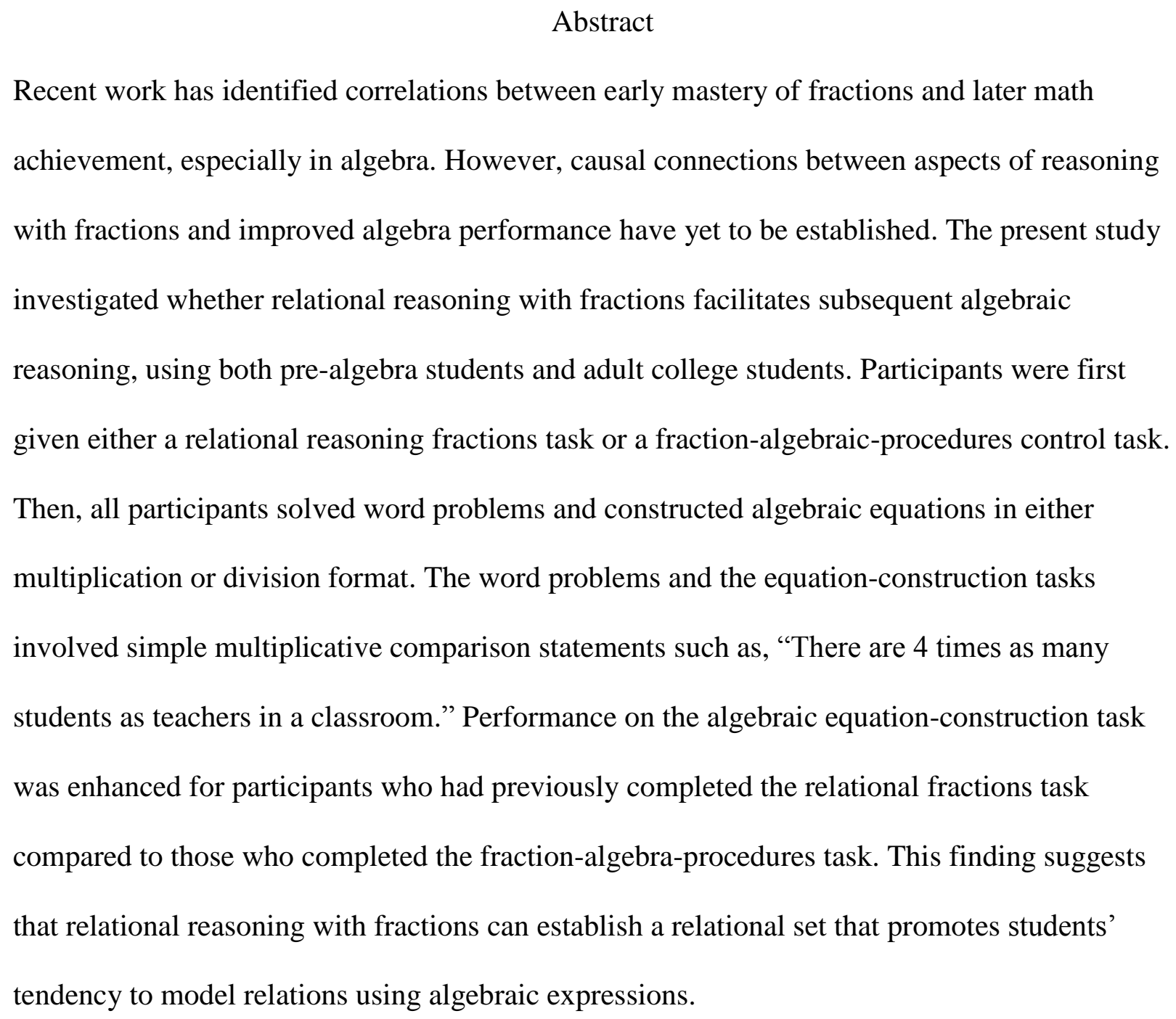


Recent research has established correlational links between early success with fractions and general math achievement (Siegler et al., 2012; Torbeyns, Schneider, Xin \& Siegler, 2015), and in particular with algebra performance (Booth \& Newton, 2012; Booth, Newton \& TwissGarity, 2014; DeWolf, Bassok \& Holyoak, 2015a; Empson \& Levi, 2011; Wu, 2001, 2009). The importance of early learning and mastery of fractions for subsequent math performance has been widely recognized, but researchers have yet to establish a more direct causal connection between specific aspects of fraction understanding and algebra performance.

Although they cannot establish causality, correlational studies have provided clues to elements of fraction understanding that are potentially important for subsequent algebra understanding. Using multiple regression analyses, DeWolf et al. (2015a) found that middleschool algebra performance was uniquely predicted by two factors: understanding of fractions as relations, and number-line estimation with decimals. In contrast, although number-line estimation with fractions and procedural knowledge about fractions in algebra equations were also significantly correlated with algebra performance, neither proved to be a unique predictor of algebra performance over and above relational fraction understanding and number-line estimation with decimals. These findings thus suggest that the relational aspect of fraction understanding, as opposed to procedural knowledge of fractions in algebra equations or facility with fraction magnitudes, has a particularly direct connection to success in algebra.

There are theoretical reasons to expect that relational understanding of fractions may support subsequent acquisition of algebra. Understanding algebra largely depends on grasping abstract relations between entities and numbers. After all, algebraic equations and expressions are meant to convey abstract relations. For example, Sfard and Linchevski (1994) have argued that success in algebra depends on students moving beyond simple mastery of how to carry out 
procedures to find solutions. Beyond procedural knowledge, students must understand that algebraic expressions convey relations between quantities, and that a general process may be used to find an unknown quantity. For example, if you have decided to split a restaurant bill between four people, but don't yet know what the total cost of the bill is $(b)$, you can express this relation as $b / 4$ or $(1 / 4) b$. This expression simultaneously represents the individual cost for each of the four people, and also a procedure that can be used to derive that specific cost depending on the actual amount of the bill.

\section{Algebraic Modeling}

One example of an area of algebra that is particularly difficult for both children and adults involves understanding and generating algebraic expressions representing multiplicative comparisons (Clement, Lochhead \& Soloway, 1979; Fisher, Borchert \& Bassok, 2011; Martin \& Bassok, 2005). For example, both children and adults have difficulty generating the correct algebraic equation for statements such as, "There are 4 times as many students as teachers in a classroom." Participants often reverse the correct order of the variables, producing $4 S=T$ rather than the correct equation $4 T=S$. This common error has generally been interpreted as reflecting direct translation of the components of the sentence (Hinsley, Hayes \& Simon, 1977; Herscovics, 1989; Fisher et. al., 2011; Graf, Bassok, Hunt \& Minstrell, 2004; Mayer \& Hegarty, 1996). Specifically, participants translate " 4 times students" as $4 S$, following the surface order in which the components (4, times, students) are mentioned in the sentence, instead of appreciating the underlying direction of the comparison between students and teachers.

This direct translation strategy appears to be a procedure used by students who do not understand how to appropriately model the relations in the equation. In addition, this strategy may also be used as a shortcut heuristic even by students who do understand algebraic modeling 
(Graf et al., 2004). Direct translation is an effective method for constructing algebraic equations in many problems (e.g., "The number of students is 6 times the number of professors"). Thus, this strategy is not always maladaptive, and in many cases can reduce cognitive load in solving the problem.

In order to distinguish genuine misconceptions about the relational structure of a problem from inappropriate use of a shortcut strategy, Fisher et al. (2011) gave participants an algebraic modeling task that required them to construct division equations as well as multiplication equations. A direct translation strategy was inapplicable to the division equations. For example, in the problem "The number of students is 6 times the number of professors," there is no word that cues division (whereas the word "times" cues multiplication). Accordingly, the correct division equation $(S / 6=P)$ can only be created by attending to the underlying mathematical relations; but unlike the case for multiplication, the surface form of the sentence does not cue a rival, erroneous equation (e.g., $P / 6=S$ ). Fisher et al. (2011) found that participants generated the correct equation much more frequently if they were asked to represent the sentence with a division equation rather than with a multiplication equation, presumably because in the absence of a direct linguistic cue to the division operator, participants are likely to think more deeply in modeling the relation.

\section{Arithmetic Word-Problem Solving}

Interestingly, performance on this algebraic modeling task (Fisher et al., 2011) is often dissociated from the processes that underlie people's solutions to arithmetic word problems (Kieran, 1992; Herscovics \& Linchevski, 1994). Martin and Bassok (2005) presented participants with matched statements or arithmetic word problems in which the same solution should be derived (e.g., "There are 3,450 students. There are 6 times as many students as 
professors. How many professors are there?"). The investigators found that participants were much more successful in solving the problems than in constructing the analogous algebraic equations. Borchert and Bassok (1998; Borchert, 2000) explicitly tested the ability of adults to transfer their correct mathematical solutions of word problems to the task of constructing algebraic equations for the relational statement in the problem text. They found that even when a word problem-solving task and the equation-construction task were presented sequentially on the same page, many adults failed to transfer their correct problem solution to the equation construction task. Conversely, even when participants constructed an incorrect equation first, they nonetheless were likely to construct a correct problem solution. This disconnection between an algebraic equation-construction task and an arithmetic problem-solving task was also evident in the written problem solutions, which never included algebraic equations.

One difference between students' understanding of arithmetic problem solving and algebra pertains to the meaning of the equal sign. In arithmetic problem solving, the equal sign is thought of as a "to-do" signal, whereas in algebra it is considered a balancing point for the two sides of the expression (Kieran, 1992). Thus, in arithmetic the emphasis is on what type of procedure needs to be executed to generate a solution (Herscovics, 1989; Stacey \& MacGregor, 2000). In algebra, by contrast, the emphasis is on identifying and expressing certain relations between numbers and quantities_-generating an actual numerical solution is secondary. The apparent lack of transfer from arithmetic word problems to algebraic equations suggests that the reasoning involved in arithmetic problem solving is largely independent from algebraic relational reasoning.

Relational reasoning with fractions may be a vehicle for improving algebraic relational reasoning. A deep conceptual understanding of fractions involves grasping that the bipartite $a / b$ 
structure of fractions conveys a relation between the numerator and denominator. This relation can take many forms, including multiplicative comparison, ratio, part-whole, probabilities, operators and quotient (Kieran, 1975, 1980; Ohlsson, 1988). Recent research has shown that adults are able to capitalize on the bipartite structure of fractions to reason about different relations between sets (DeWolf, Bassok \& Holyoak, 2015b; Lee, DeWolf, Bassok \& Holyoak, 2016; Rapp, Bassok, DeWolf \& Holyoak, 2015). For example, DeWolf et al. (2015b) asked participants to identify part-to-part ratios and part-to-whole ratios between two subsets in a display, denoted by either a decimal ratio or fraction ratio. When participants were shown decimals, which do not provide explicit relational information, performance was far less accurate than when they were shown the corresponding fractions. This finding suggests that successful relation discrimination is facilitated by fractions, which can be decomposed into two relational components, as compared to decimals, which lack a similar type of internal relational structure. That is, the fraction components serve to explicate the quantities that enter into a relation. Importantly, understanding fractions as relations does not require calculating a quotient (much like understanding algebra expressions such as $b / 4$, for which the magnitude is not immediately calculable).

\section{Present Study}

The goal of the current study was to test whether relational thinking about fractions can help middle-school pre-algebra students and college-level adults to construct correct division or multiplication algebraic equations that represent simple multiplicative-comparison statements. We sought to go beyond previous correlational studies by attempting to determine whether relational understanding of fractions has a causal connection to algebra performance. More specifically, we assessed participants' algebra performance (i.e., equation-construction task) 
immediately after they had performed a task designed to activate relational knowledge of fractions, or a control task that focused on the procedures used to solve algebra problems containing fractions. In order to compare the effect of the fraction relations task against a baseline for algebra performance, we selected a control task that had previously been found to be unrelated to algebra performance (Booth et al., 2014; DeWolf et al., 2015a). The basic aim was to determine whether relational reasoning with fractions would establish a general relational set, which would in turn enhance relational reasoning with fractions (especially in the case of division equations, for which a direct-translation strategy is not possible). Studies of nonmathematical reasoning have shown similar transfer effects based on establishing a relational set (Chaxel, 2015; Vendetti, Wu \& Holyoak, 2014).

In addition to participants drawn from middle school pre-algebra classes, we also included college students in the study. If a relatively high level of overall math expertise is required to allow transfer at the level of a relational set, then such transfer may only be observed for adults. But if a grasp of the relational nature of fractions is sufficient, then even middleschool students may show relation-based transfer from fractions to algebra.

We also assessed whether relational reasoning with fractions would enhance performance on arithmetic word problems. Given the apparent disconnect between algebraic problem solving (which relies more on abstract reasoning) and solving of arithmetic word problems (which depends on execution of a set of procedures), it seems possible that relational reasoning with fractions may fail to enhance solution of word problems, even if it promotes successful construction of algebraic equations. 


\section{Method}

\section{Participants}

Participants were drawn from two separate sets of child and adult populations. The children were students enrolled in introductory pre-algebra courses from a local Los Angeles school. A total of 101 seventh-grade middle-school students (mean age $=12.5, S D=.54 ; 48$ male, 53 female) participated in the study near the end of the school year. Students were not compensated for participating. Adults were recruited from undergraduate psychology courses at the University of California, Los Angeles. A total of 114 students (mean age $=20.1, S D=1.59 ; 2$ male, 93 female) participated. UCLA students received one course credit to fulfill a study requirement for their undergraduate course.

\section{Materials and Design}

The study included two dependent measures (word problem-solving task, and algebra equation-construction task), which were analyzed separately. For equation-construction task, the design was a 2 (age group: $7^{\text {th }}$ graders vs. college students) X 2 (initial task task: fraction relations vs. fraction-algebra procedures) X 2 (algebra equation format: division vs. multiplication). The design for the word problem-solving task was the same, except that the variable of equation format was omitted. All tasks are described in more detail below. ${ }^{1}$

Fraction-relations task. The fraction relations task consisted of six problems (see Appendix A for a reproduction of the task). This task was adapted from a similar task that showed high levels of performance when used previously with adults (DeWolf et al., 2015b). The problems contained displays of two discrete sets. Participants were instructed to create a fraction that either highlighted the ratio of one set to the other (Part-Part Ratio, PPR) or the ratio of one part to the whole set (Part-Whole Ratio, PWR; see Figure 1 for an example). The first two 
problems consisted of one PPR problem and one PWR problem. Each of these problems included feedback. Participants were given a chance to provide an answer on the first page of the problem. The second page provided a detailed explanation of the answer and a visual diagram depicting the differences between the subsets. The six problems appeared in one of three random orders (where the first two were always a PPR and PWR problem with feedback).

Insert Figure 1 about here

Fraction-algebra-procedures control task. The fraction-algebra-procedures task consisted of six problems adapted from a previous algebra problem set designed to test understanding of basic algebraic procedures (Booth et al., 2014; DeWolf et al., 2015a). The questions focused on understanding of how to manipulate an algebra equation that contained a fraction. Appendix A provides examples of the problems. These problems tested basic knowledge about steps to complete algebra problems. Previous research (Booth et al., 2014; DeWolf et al., 2015a) found that performance on this task is unrelated to algebra performance. Therefore, we did not expect that performing this task would benefit the subsequent algebraic modeling task. Rather, this task was selected to provide a control task that equated the amount of time participants spent in performing an initial task. We chose a math task and not a completely unrelated task (e.g., a crossword puzzle) in order to avoid potential task switching costs, as participants completed this task directly before the algebra tasks. The problems appeared in one of three random orders. In order to ensure this condition served a true control for time on task (rather than a learning opportunity), no feedback was provided for any of the problems. 
Word problem-solving task. Following the initial task, all participants were given the same two word problems to complete: "There are 72 athletes in a league. There are 4 times as many athletes as coaches. How many coaches are there?" and, "One day at a bakery, 64 people ordered cupcakes. 4 times as many people ordered cupcakes as muffins. How many people ordered muffins?" The word problems mirrored the multiplicative comparison statements given later on in the algebra equation construction task. Participants were asked to show their work and were given space to write their answers. The order of the problems was counterbalanced across participants.

Algebra equation-construction task. These materials were adapted from those used by Fisher et al. (2011). Participants were given detailed instructions on how to create algebraic equations for the four basic arithmetic operations (see Appendix A). They received an example using an addition problem, and then as practice were asked to create a subtraction equation based on a new scenario. Participants were then given four different multiplicative comparison statements. Each statement appeared on a separate page. For two of them, participants had to generate a division equation to represent the statement; for the other two, they had to generate a multiplication equation to represent the statement. For example, one statement was, "In a certain classroom, there are 4 times as many students as teachers." An example of a division equation would be, " $s / 4=t$ ". The corresponding multiplication equation would be, " $4 * t=s$ ". The assignment of division or multiplication as the operation was counterbalanced across the four problem scenarios. The statements themselves appeared in one of three different random orders across participants.

\section{Procedure}


All participants completed the set of tasks using paper and pencil, administered in packets. They were instructed to proceed through the packet in order and not return to any pages. Participants were encouraged to show their thinking or their work throughout the packet. Participants first completed either the fraction relations task or the algebra procedures control task. Directly after, they completed the word problem-solving task, and finally the algebra equation-construction task. For consistency, each of the two age groups $\left(7^{\text {th }}\right.$ graders and college students) completed the exact same packets and materials. The $7^{\text {th }}$ graders had a maximum of 50 minutes to complete the packet, though most took about 25 minutes. College students completed the packets in about 10 minutes on average. There was no time limit for the college students, but they were encouraged to try to go as quickly as possible while not sacrificing accuracy.

\section{Results}

\section{Initial Task Performance}

Questions for each initial task were scored for accuracy on a binary $(0,1)$ basis. Figure $2 \mathrm{a}$ displays the mean performance for each task. A 2 (age group) X 2 (fraction relations vs. fractions-algebra procedures task) between-subjects ANOVA revealed a significant main effect of age group (adults: $91 \%$ vs. children $66 \%, F(1,211)=137.59, M S E=.025, p<.001, \eta_{p}{ }^{2}=$ .39), moderated by a significant interaction between the two factors $(F(1,211)=65.23, M S E=$ $\left..025, p<.001, \eta_{p}{ }^{2}=.24\right)$. Planned comparisons revealed that the $7^{\text {th }}$ graders performed significantly more accurately on the fraction relations (FR) task compared to the fractionalgebraic procedures (FAP) task (FR: 85\% vs. FAP: 47\%; $t(99)=9.76, p<.001, d=1.94)$, whereas there was no difference in performance between the two tasks for college students (FR: $93 \%$ vs. FAP: $90 \% ; t(111)=1.26, p=.21, d=.26)$. Word Problem-Solving Task 
Each of the two word problems was scored for accuracy on a binary 0, 1 basis.

Participants needed to give the correct numerical answer to receive credit on each question.

Figure $2 \mathrm{~b}$ displays mean performance on this task. A 2 (age group) X 2 (FR vs. FAP task)

between-subjects ANOVA $^{2}$ revealed no significant difference between accuracy after performing the FR task $(70 \%)$ compared to the FAP task $\left(67 \% ; F(1,211)=.78, p=.39, \eta_{p}{ }^{2}=.004\right)$, nor was there a significant interaction between initial task and age $(F(1,211)=.71, M S E=.15, p=.40$, $\left.\eta_{p}{ }^{2}=.005\right)$. The college students performed significantly higher than the $7^{\text {th }}$ graders (adults: $90 \%$ vs. children: $\left.47 \% ; F(1,211)=66.43, M S E=.15, p<.001, \eta_{p}{ }^{2}=.26\right)$.

In order to assess whether participants used algebra in solving the word problems, we coded spontaneous generation of algebra equations. If a participant wrote down an algebra equation when solving the problem (either correct or incorrect), that problem was coded as a 1 . If the participant used only the numbers in the problem and no variables to represent the abstract relation, that problem was coded as a 0 . A 2 (age group) X 2 (initial task) between-subjects ANOVA revealed an interaction $\left(F(1,211)=3.79, M S E=.16, p=.05, \eta_{p}{ }^{2}=.02\right)$. Initial task did not impact use of equations for the $7^{\text {th }}$ graders (FR: $8 \%$ vs. FAP: $8 \%, t(99)=.03, p=.98, d=$ .007). However, the rate of spontaneously generating algebra equations when solving the word problems was higher for adults who completed the FR task compared to those who completed the FAP task (FR: $51 \%$ vs. FAP: $30 \%, t(112)=2.33, p=.02, d=.44$ ). This finding suggests that, at least for adults, completing the fraction relations task first increased the likelihood of thinking about word problem in terms of algebraic relationships. However, generating algebra equations did not lead to greater success in solving the word problems even for adults, perhaps because their performance was near ceiling on this task. 


\section{Algebra Equation-Construction Performance}

Each algebra equation was scored on a $(0,1)$ binary basis for accuracy. Figure $2 c$ displays the mean accuracies for each equation condition by age group. A 2 (equation type: division vs. multiplication) X 2 (initial task) X 2 (age group) mixed ANOVA revealed a significant 2-way interaction between equation type and initial task $\left(F(1,211)=4.6, M S E=.097, p=.03, \eta_{p}{ }^{2}=\right.$ .02), such that participants performed better on division equations compared to multiplication equations after completing the FR task (division: $78 \%$ vs. multiplication: $63 \%, t(107)=3.3, p=$ $.001, d=.33)$, whereas there was no difference in performance between division and multiplication after completing the FAP task (division: $61 \%$ vs. multiplication: $60 \%, t(106)=$ $.32, p=.75, d=.03)$. Similarly, completing the FR task resulted in higher performance on the division equations relative to completing the FAP control task (FR: 78\% vs. FAP: $62 \%$; $t(213)=$ $2.99, p=.003, d=.40$ ), whereas there was no difference in performance on the multiplication equations between the two initial tasks (FR: 64\% vs. FAP: $61 \% ; t(213)=.58, p=.56, d=.07$ ).

There was also a significant 2-way interaction between equation type and age group $(F(1$, $\left.211)=5.73, M S E=.097, p=.02, \eta_{p}^{2}=.03\right)$, such that the $7^{\text {th }}$ graders performed better on division equations (division: $55 \%$ vs. multiplication: $40 \%, t(100)=2.75, p=.007, d=.35$ ), whereas there was no difference in performance for college students (division: $84 \%$ vs. multiplication: $83 \%, t(113)=.31, p=.76, d=.03)$. However, there was no significant 2-way interaction between initial task and age group $\left(F(1,211)=.02, M S E=.20, p=.90, \eta_{p}{ }^{2}<.001\right)$, consistent with college students achieving superior performance compared to the $7^{\text {th }}$ graders after completing either the FR or FAP task. The 3-way interaction was not reliable $(F(1,211)=1.61$, $\left.M S E=.10, p=.21, \eta_{p}{ }^{2}=.008\right)$. Together, these findings show that completing the fraction relations task improved subsequent performance on the algebra equation-construction task 
compared to the control task, with this facilitation being especially strong for $7^{\text {th }}$ graders' construction of division equations.

\section{Discussion}

The goal of the present study was to investigate the extent to which relational reasoning with fractions plays a causal role in enhancing subsequent creation of algebraic equations. Participants were asked to complete either a task focused on fraction relations or a control task using fraction algebra procedures. The results showed that both $7^{\text {th }}$ graders and college students were more accurate on an algebra equation-construction task after completing the fraction relations task, as compared to completing the control task. The positive impact of the fraction relations task was especially strong for $7^{\text {th }}$ graders when creating division equations in the target algebraic modeling task.

Previous research on the algebraic equation-construction task suggests that requiring creation of a division (rather than a multiplication) equation blocks reliance on the directtranslation heuristic (e.g., multiplication cued by the word "times"), and encourages modeling of the relational structure (Fisher et al., 2011). Here, we found that performing the fraction relations task helped participants to engage in the abstract thinking required for constructing correct algebraic division equations to represent multiplicative comparison statements. This finding supports our hypothesis that solving fraction relation problems encourages a general relational set, which facilitates representing object relations with algebraic equations because the latter task involves the same type of relational reasoning.

For the $7^{\text {th }}$ graders, performance on the fraction relations task was much better than on the algebra-procedures control task. The latter task focused on small procedural details of solving algebraic equations and was most likely beyond the capability of many of the $7^{\text {th }}$ grade students. 
By comparison, the fraction relations task was very simple and straightforward. Interestingly, even though adults performed both the fraction relations and algebra procedures tasks very well, only the fraction relations task yielded a significant improvement in constructing algebraic equations. Thus, sheer difficulty of the initial task did does not seem to be the critical factor in enhancing performance on the equation-construction task.

Consistent with prior findings revealing a disconnection between arithmetic and algebra (Kieran, 1992; Herscovics \& Linchevski, 1994), the fraction relations task did not affect accuracy in solving word problems involving multiplicative-comparison statements. The $7^{\text {th }}$ graders seemed to find the task of solving word problems generally difficult, yielding low accuracy rates. College students, in contrast, were near ceiling on this task. For college students, completing the fraction relations task (compared to the control task) increased the spontaneous rate of using algebraic equations in the word problem task. For the $7^{\text {th }}$ graders, using algebra equations to solve word problems was extremely rare, with only $8 \%$ of responses including algebra equations.

The task of identifying relations represented by fractions highlights the flexibility of the notational structure of a fraction. In this task, participants learn to focus on the relations between the numbers rather than on calculating any specific value. The focus is on the different types of ratios or proportions that can be represented using the fraction notation. Proportionality, though usually taught later in school and separately from basic fraction lessons, is often taught as one of the first examples of algebraic problem solving (Ohlsson, 1998). For example, students are given problems like, “There are 6 apples for every 8 oranges. If we have 10 apples, how many oranges do we have?" The basic set up for such a problem is something like, $\frac{6 \text { apples }}{8 \text { oranges }}=\frac{10 \text { apples }}{\text { x oranges }}$. Students learn to express an unknown with a variable (" $x$ "), and how to solve the proportion. 
Thus, students are taught that the actual integrated value of the proportion is secondary, and learn to focus instead on the relations between corresponding values. The relation identification task used in the present study taps into a similar type of thinking about fractions, shifting attention and focus to the relational structure and away from calculations.

In the current study, we used only six problems in the initial task and only four algebra equation-construction questions. Future research is needed to determine whether there are other types of algebraic tasks that could benefit from relational fraction training, and whether the benefits of such training persist over time. Perhaps relational fractions training could be developed into a more formal intervention to foster students' understanding of both fractions and algebra. Future research should also examine how relational fraction understanding develops as students progress through introductory to mastery levels of algebra, and at what point this type of training would be the most useful. In addition, $7^{\text {th }}$ grader's performance on the FAP task was substantially lower than performance on the FR task. Future research is needed to determine whether there are other types of fraction tasks that might benefit different aspects of algebra. In summary, the present study provides evidence that relational reasoning required to successfully generate algebraic equations can be facilitated by a task that requires relational reasoning with fractions. This finding supports the hypothesis that fractions provide an important conceptual foundation for understanding mathematical expressions, and that relational reasoning with fractions plays a causal role in promoting relational reasoning about algebraic problems. This basic idea has also been emphasized by Empson and her colleagues (Empson, 1999; Empson \& Levi, 2011; Empson, Levi \& Carpenter, 2011), who have argued that core intuitions about relations between numbers, first established in whole-number arithmetic and later emphasized with basic fraction concepts, provide the building blocks for abstract relational 
expressions in algebra. In general, it seems that a sequence of instruction (typical in American curricula) that introduces algebraic word problems in relation to simpler non-algebraic word problems does not highlight the relational nature of algebraic expressions. Our findings suggest that using fractions to emphasize relational expressions may encourage a better transition from a focus on calculations to a focus on abstract models. The usefulness of fractions for learning algebra seems to be based on appreciating the value of a relational expression. 


\section{Footnotes}

${ }^{1}$ The tasks chosen for this experiment were selected and adapted from previous research (DeWolf et al., 2015a, 2015b; Fisher et al., 2011; Martin \& Bassok, 2005). In previous research, these tasks showed high internal reliability within subjects. In this study, both the FAP (Cronbach's Alpha: .81) and the FR (Cronbach's Alpha: .72) tasks had high reliability.

${ }^{2}$ In parallel with this and all subsequent ANOVA analyses, we also used a non-parametric sign test to verify whether the results held (given the bounded nature of our outcome measures for the word problem solving and algebra equation tasks). These non-parametric tests showed the same pattern of results as the ANOVA analyses reported here. 


\section{Acknowledgements}

Preparation of this paper was supported by NSF Fellowship DGE-1144087 to MD. We thank

Austin Chau for help with collecting data. A portion of the data was presented at the $9^{\text {th }}$ Biennial

Meeting of the Cognitive Development Society in Columbus, Ohio (October, 2015). 


\section{References}

Booth, J. L., \& Newton, K. J. (2012). Fractions: Could they really be the gatekeeper's doorman? Contemporary Educational Psychology, 37, 247-253.

Booth, J. L., Newton, K. J., \& Twiss-Garrity, L. K. (2014). The impact of fraction magnitude knowledge on algebra performance and learning. Journal of Experimental Child Psychology, $118,110-118$.

Borchert, K., \& Bassok, M. (1998). Wor(l)d problems: World knowledge and mathematics. Paper presented at the annual research meeting of the Department of Psychology, University of Washington, Seattle.

Borchert, K. (2000). Connecting operational and structural knowledge in algebra: The impact of word problem solving on equation construction. Unpublished Master's thesis, Department of Psychology, University of Washington.

Chaxel, A.-S. (2015). The impact of a relational mindset on information distortion. Journal of Experimental Social Psychology, 60, 1-7.

DeWolf, M., Bassok, M., \& Holyoak, K. J. (2015a). From rational numbers to algebra: Separable contributions of decimal magnitude and relational understanding of fractions. Journal of Experimental Child Psychology, 133, $72-84$.

DeWolf, M., Bassok, M., \& Holyoak, K. J. (2015b). Conceptual structure and the procedural affordances of rational numbers: Relational reasoning with fractions and decimals. Journal of Experimental Psychology: General, 144, 127-150.

Empson, S. B. (1999). Equal sharing and shared meaning: The development of fraction concepts in a first-grade classroom. Cognition and Instruction, 17, 283-342. 
Empson, S. B., \& Levi, L. (2011). Extending children's mathematics: Fractions and decimals. Portsmouth, NH: Heinemann.

Empson, S. B., Levi, L., \& Carpenter, T. P. (2011). The algebraic nature of fractions: Developing relational thinking in elementary school. In J. Cai \& E. Knuth (Eds.), Early algebraization: A global dialogue from multiple perspectives (pp. 409-428). Berlin: Springer.

Fisher, K. J., Borchert, K., \& Bassok, M. (2011). Following the standard form: Effects of equation format on algebraic modeling. Memory \& Cognition, 39(3), 502-515.

Graf, E. A., Bassok, M., Hunt, E., \& Minstrell, J. (2004). A computer-based tutorial for algebraic representation: The effects of scaffolding on performance during the tutorial and on a transfer task. Technology Instruction Cognition and Learning, 2, 135-170.

Herscovics, N. (1989). Cognitive obstacles encountered in the learning of algebra. In S. Wagner \& C. Kieran (Eds.), Research issues in the learning and teaching of algebra (pp. 60-86). Hillsdale, NJ: Erlbaum.

Herscovics, N., \& Linchevski, L. (1994). A cognitive gap between arithmetic and algebra. Educational Studies in Mathematics, 27, 59-78.

Hinsley, D. A., Hayes, J. R., \& Simon, H. A. (1977). From words to equations: Meaning and representation in algebra word problems. In M. A. Just \& P. A. Carpenter (Eds.), Cognitive processes in comprehension (pp. 89-106). Hillsdale, NJ: Erlbaum.

Kieren, T. (1975). On the mathematical, cognitive, and instructional foundations of rational numbers. In R. Lesh (Ed.), Number and measurement: Papers from a research workshop (pp. 101-144). Columbus, OH: ERIC/SMEAC.

Kieren, T. (1980). The rational number construct- its elements and mechanisms. In T. E. Kieren (Ed.), Recent research on number learning (pp. 125-149). Columbus, OH: ERIC/AMEAC. 
Kieran, C. (1992). The learning and teaching of school algebra. In D. A. Grouws (Ed.), Handbook of research on mathematics teaching and learning (pp. 390-419). New York: Macmillan.

Lee, H. S., DeWolf, M., Bassok, M., \& Holyoak, K. J. (2016). Conceptual and procedural distinctions between fractions and decimals: A cross-national comparison. Cognition, 147, $57-69$.

Martin, S. A., \& Bassok, M. (2005). Effects of semantic cues on mathematical modeling: evidence from word-problem solving and equation construction tasks. Memory \& Cognition, $33(3), 471-8$.

Ohlsson S. (1988). Mathematical meaning and applicational meaning in the semantics of fractions and related concepts. In J. Hiebert \& M. Behr (Eds.) Number concepts and operations in the middle grades (Vol. 2, pp. 53-92). Reston, VA: Erlbaum \& National Council of Teachers of Mathematics.

Rapp, M., Bassok, M., DeWolf, M., \& Holyoak, K. J. (2015). Modeling discrete and continuous entities with fractions and decimals, Journal of Experimental Psychology: Applied, 21(1), 4756.

Sfard, A., \& Linchevski, L. (1994). The gains and the pitfalls of reification: The case of algebra. Educational Studies in Mathematics, 26, 191-228.

Siegler, R. S., Duncan, G. J., Davis-Kean, P. E., Duckworth, K., Claessens, A., Engel, M., et al. (2012). Early predictors of high school mathematics achievement. Psychological Science, 23, 691-697.

Stacey, K., \& MacGregor, M. (1999). Learning the algebraic method of solving problems. Journal of Mathematical Behavior, 18,149-167. 
Torbeyns, J., Schneider, M., Xin, Z., \& Siegler, R. S. (2015). Bridging the gap: Fraction understanding is central to mathematics achievement in students from three different continents. Learning and Instruction, 37, 5-13.

Vendetti, M. S., Wu, A., \& Holyoak, K. J. (2014). Far-out thinking: Generating solutions to distant analogies promotes relational thinking. Psychological Science, 25, 928-933.

Wu, H. (2001). How to prepare students for algebra. American Educator, 25(2), 10-17.

Wu, H. (2009). What's sophisticated about elementary mathematics? American Educator, 33(3), 4-14. 
Figures

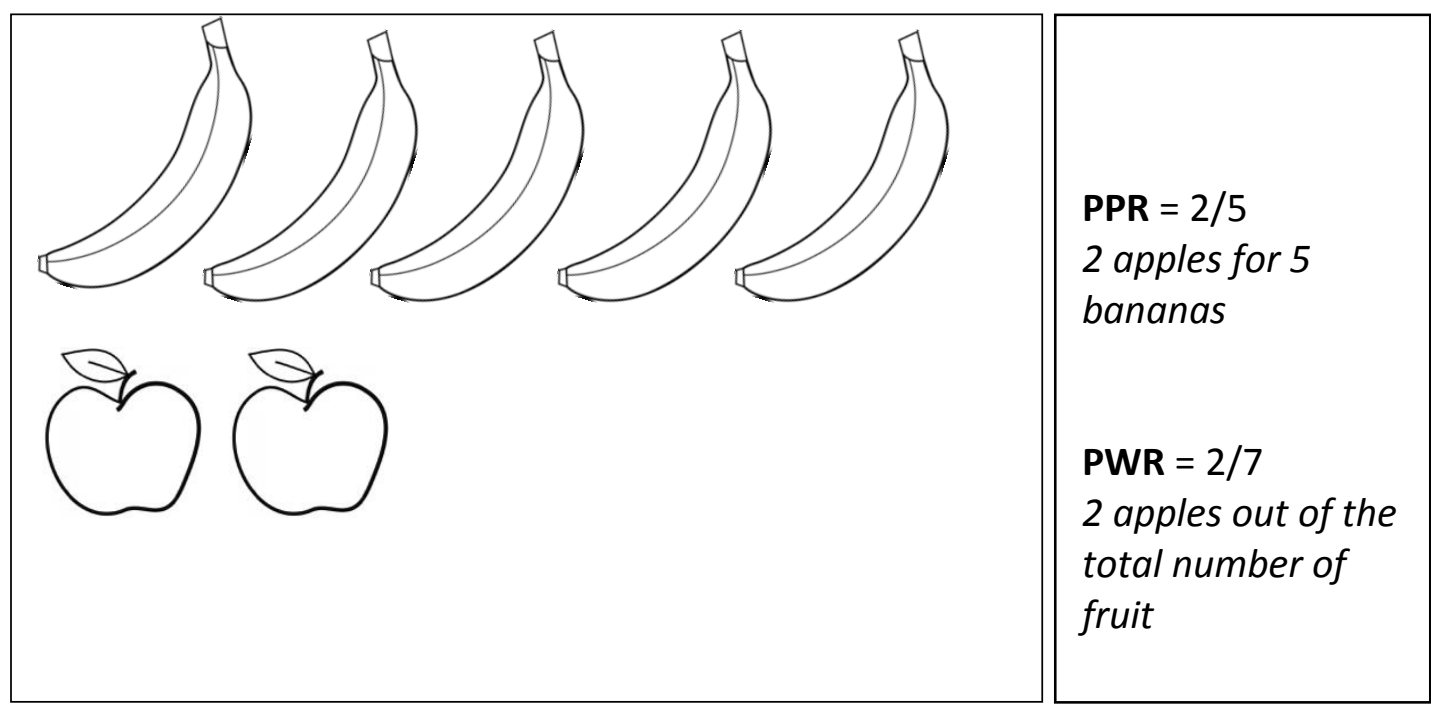

Figure 1. Example of stimuli used in the fraction relations task. A part-to-part ratio (PPR) would be $2 / 5$, denoting the ratio of one subset to another subset; and a part-to-whole ratio (PWR) would be $2 / 7$, denoting the ratio of one subset to the set. 


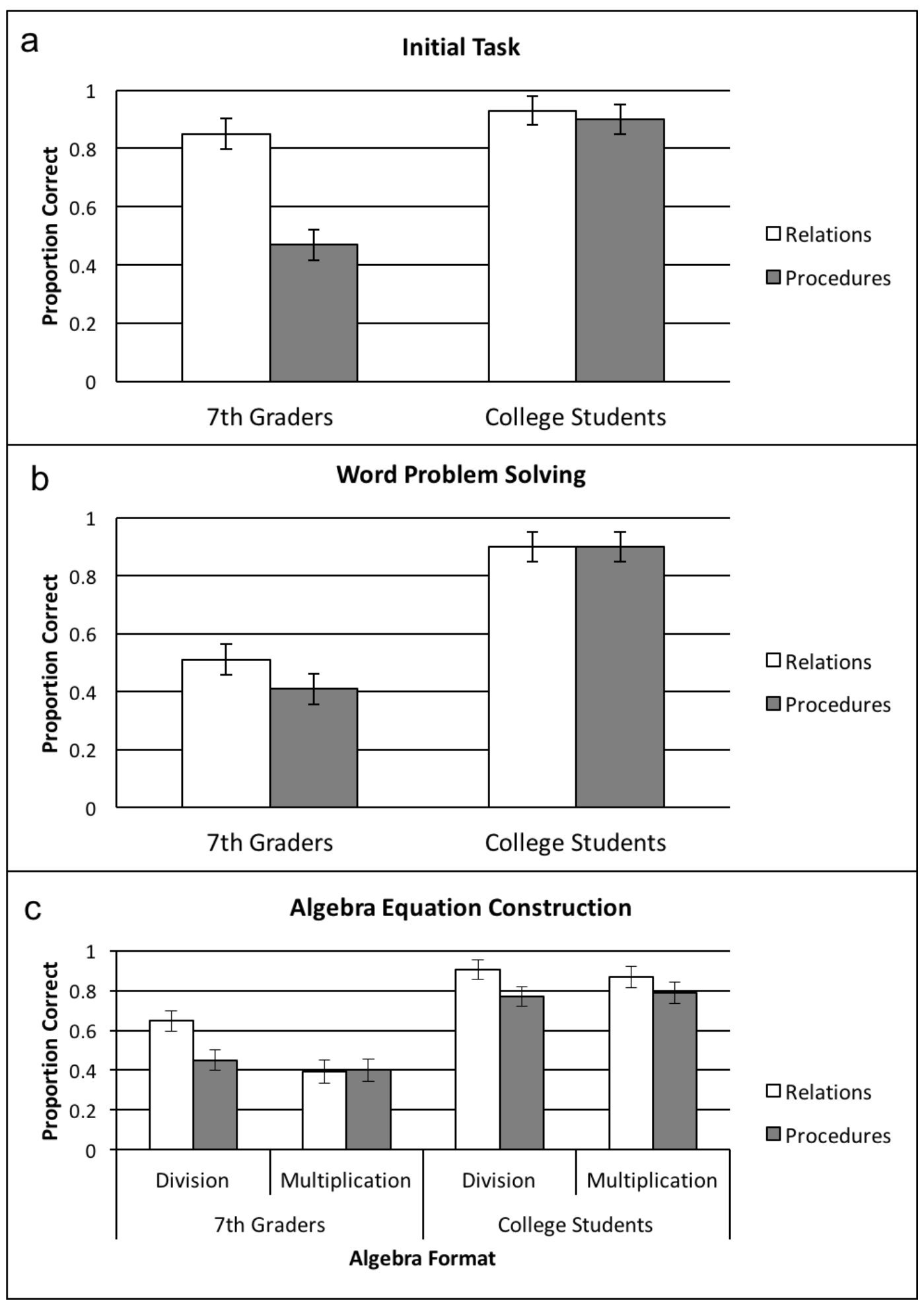

Figure 2. Proportion correct for the initial task (fraction relations vs. fraction-algebraic procedures; panel a), the word problem-solving task (panel b) and the algebra equationconstruction task (panel c) by age of participants. 
DeWolf et al.

Appendix A: Materials

Fraction Relations Task:

In the box below, there are some flowers: roses and daisies.

What part of the flowers are the roses?

(hint: roses are white in the picture)

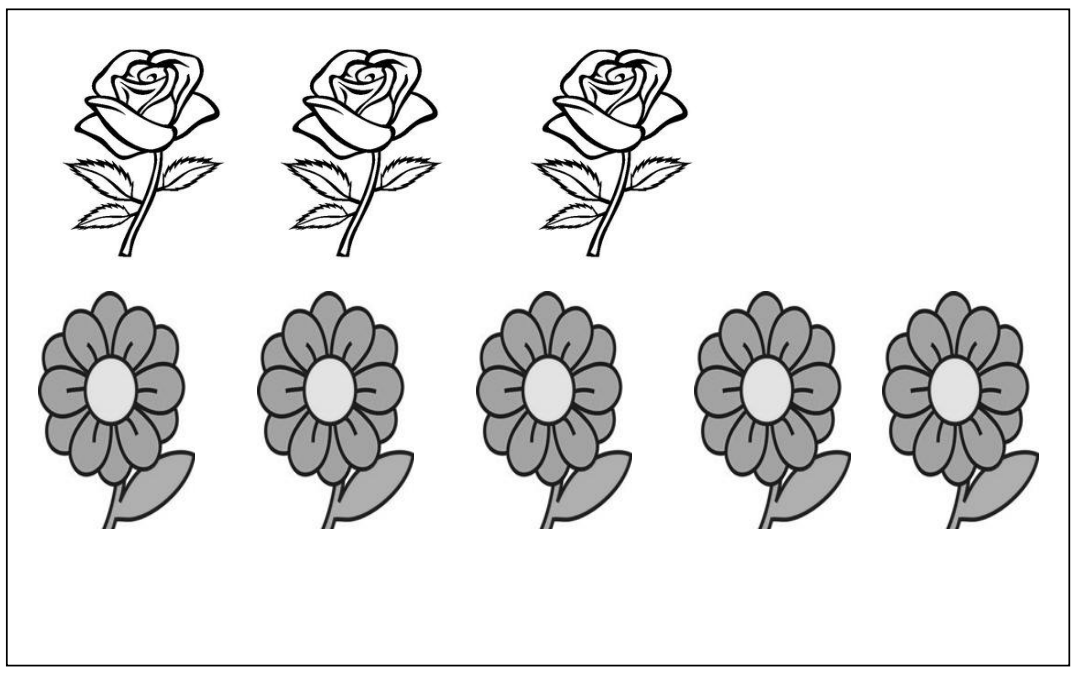

Answer: 
DeWolf et al.

Here is how to find the answer:

There are $\underline{3}$ roses

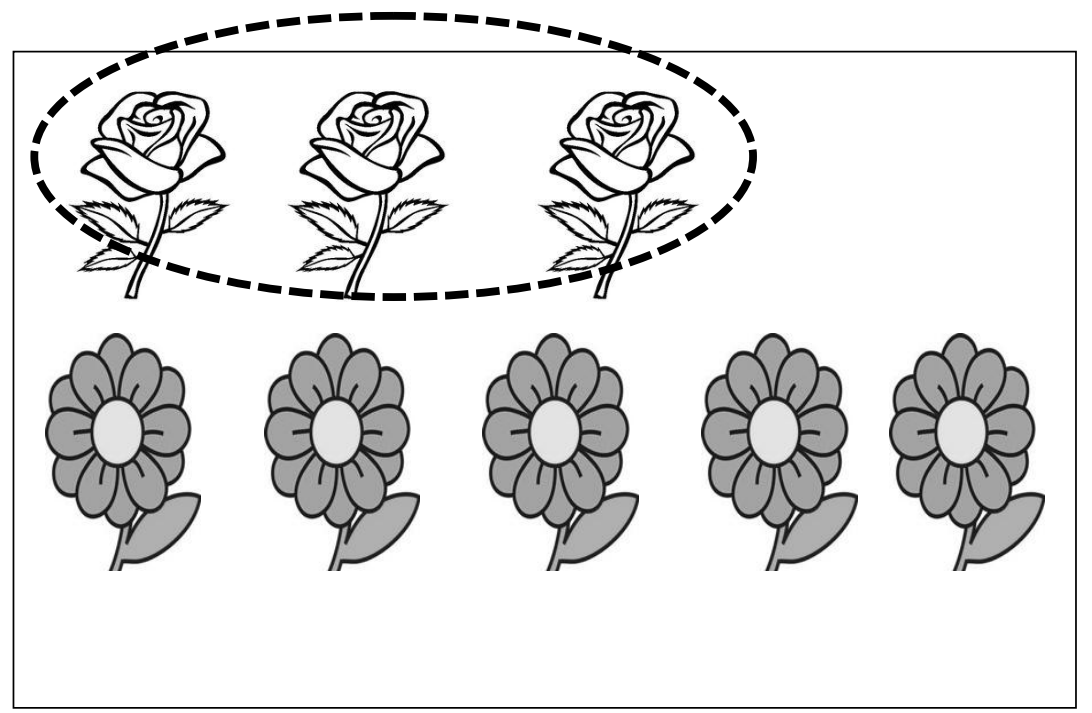

There are $\underline{8}$ total flowers

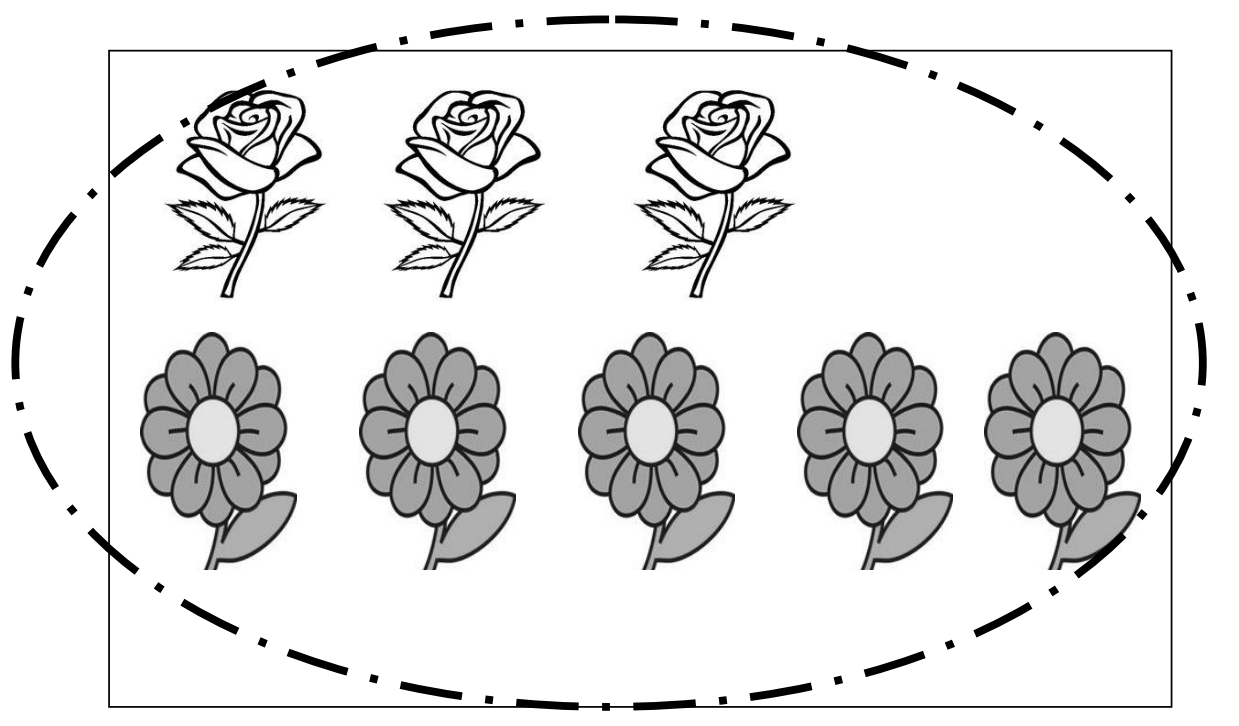

So, the roses are $\mathbf{3} / \mathbf{8}$ of the flowers 
In the box below, there are some children: girls and boys.

What is the ratio of girls to boys?

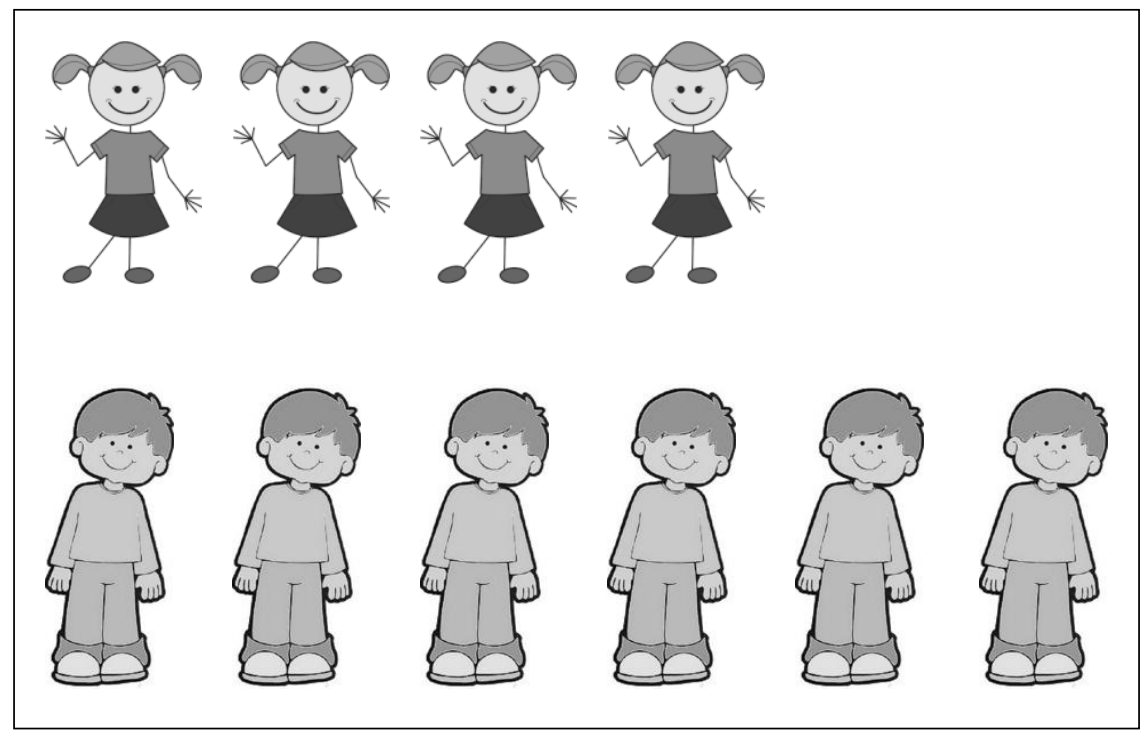

Answer: 
Here's how to find the answer:

There are $\mathbf{4}$ girls

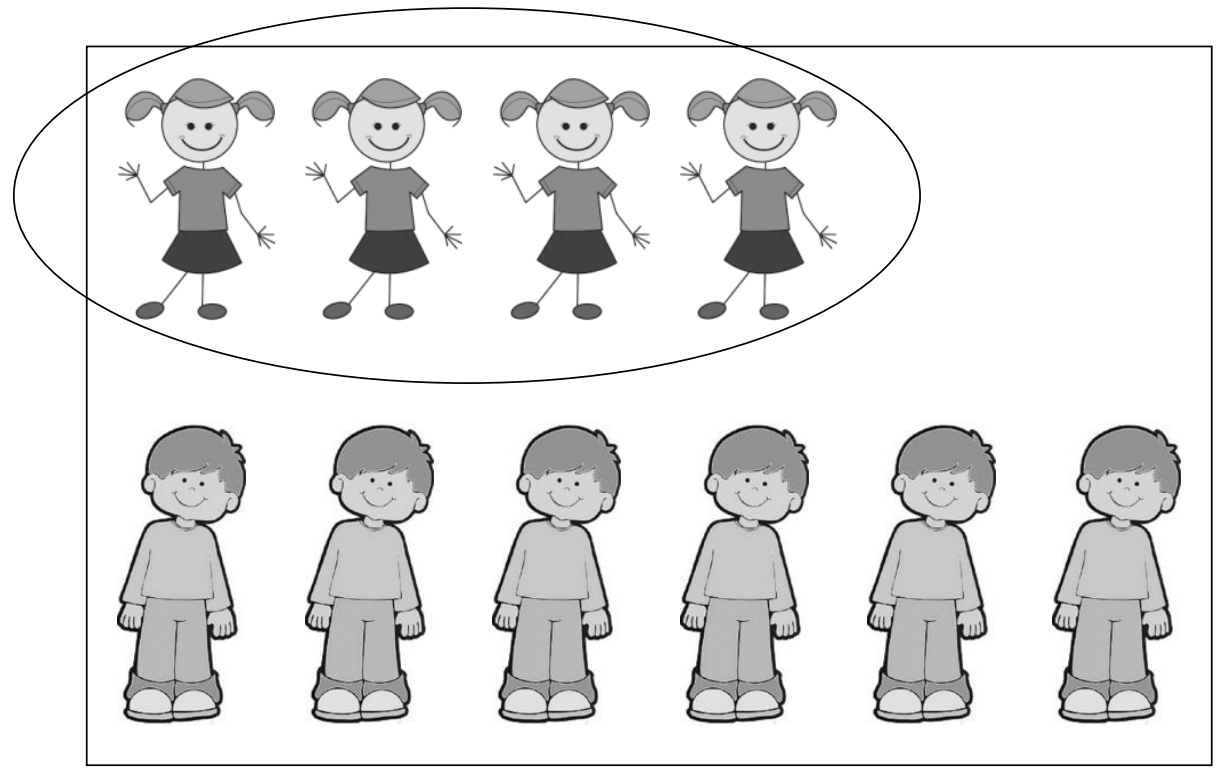

There are 6 boys

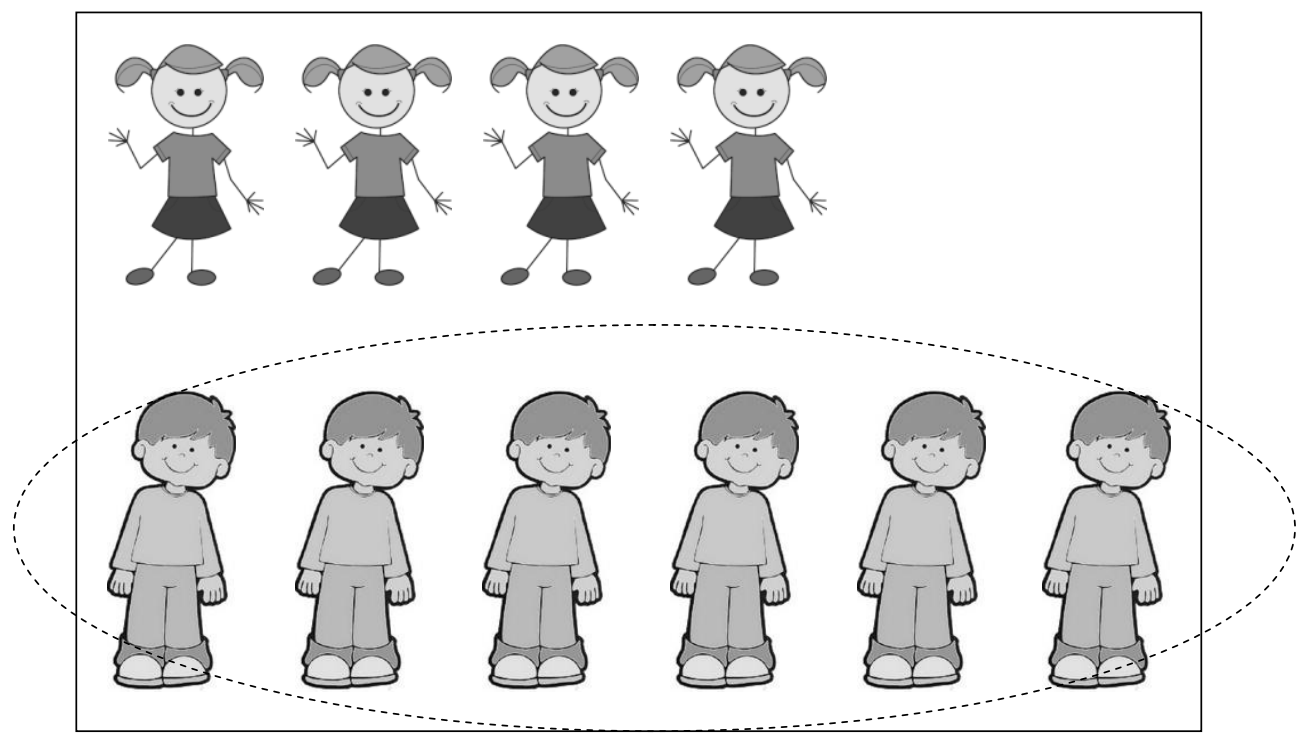

So the ratio of girls to boys is $4 / 6$ 
In the box below there are some pieces of fruit: bananas and apples.

What part of the total pieces of fruit are the bananas?

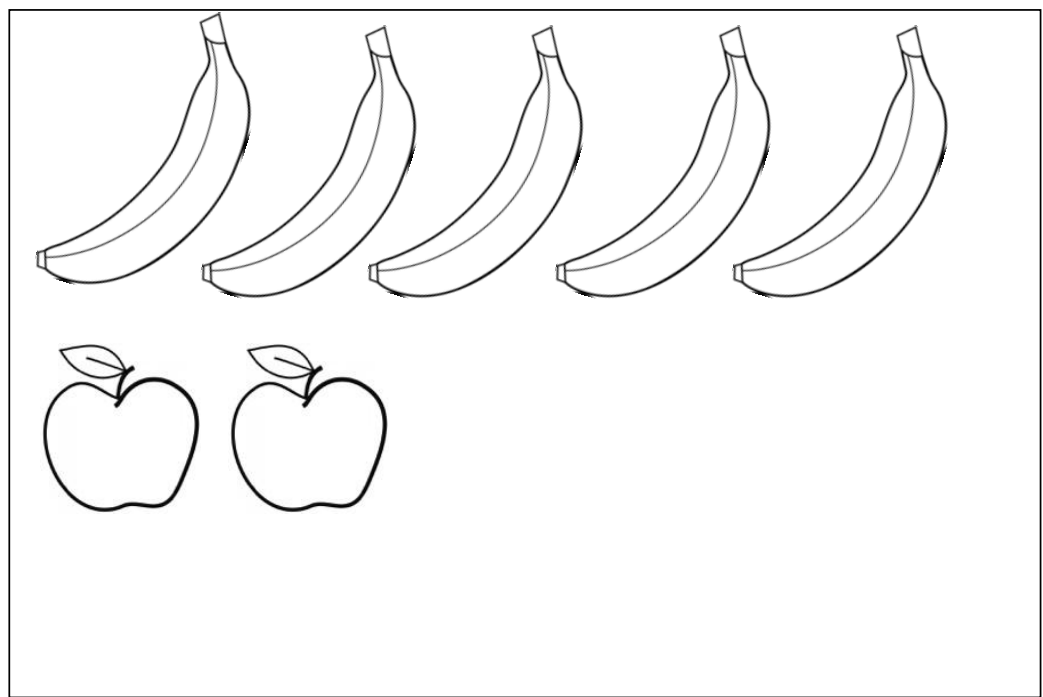

Answer:

In the box below, there are some pieces of fruit: strawberries and pears. What part out of the total pieces of fruit are the strawberries?

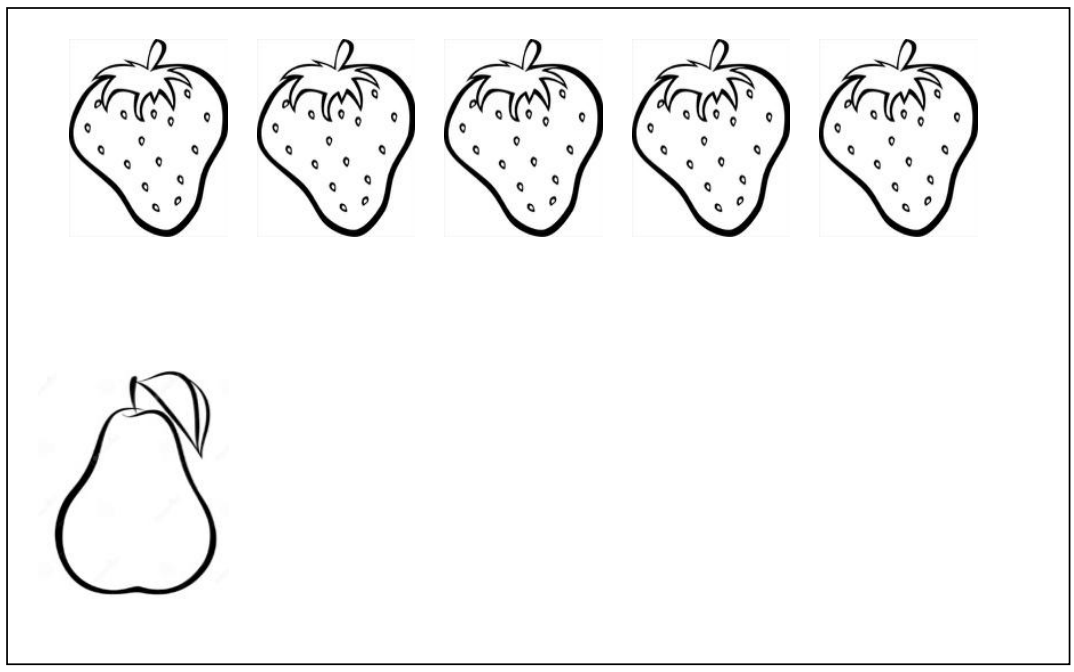

Answer: 
In the box below, there are some desserts: brownies and cookies.

What is the ratio of cookies to brownies?

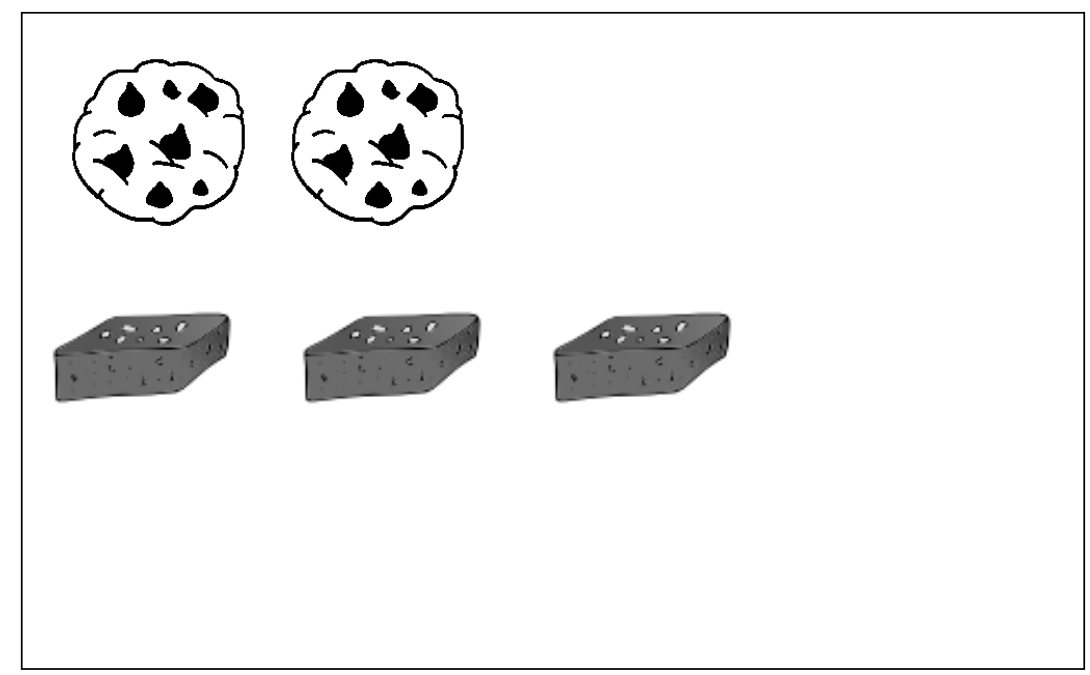

Answer:

In the box below, there are writing instruments: crayons and pencils.

What is the ratio of crayons to pencils?

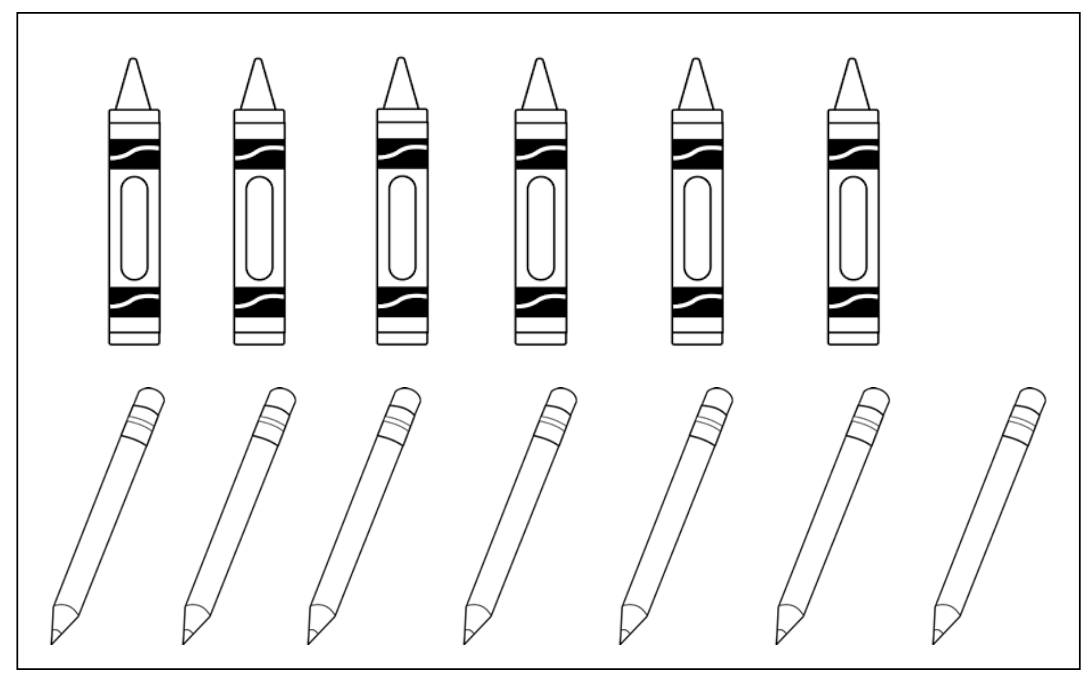

Answer: 


\section{Algebra Procedures Task:}

Please answer the following questions:

1. Are either of these an effective first step toward solving for $z$ in the equation $3=\frac{1}{z}$ ? Circle yes or no.
a. Multiply both sides by $z$.
Yes No
b. Divide both sides by 3 .
Yes No

2. Would any of the following steps be an effective first step toward solving the equation $\frac{6}{d}=2$ ? Circle yes or no.
a. Subtract 2 from both sides.
Yes No
b. Multiply both sides by $d$.
Yes No

3. If $y=3 x+2$, which of these expresses $x$ in terms of $y$ ? Circle the correct answer.
$x=\frac{y-2}{3}$
b. $x=\frac{y+2}{3}$
c. $x=\frac{y}{3}-2$
d. $x=\frac{y}{3}+2$

4. Are either of these an effective first step toward solving for $y$ in the equation $\frac{1}{4}+y=10$ ? Circle yes or no.
a. Multiply both sides by $y$
Yes No
b. Subtract both sides by $\frac{1}{4}$
Yes No

5. Would any of the following steps be an effective first step toward solving the equation: $5+x=\frac{2}{3}$ ? Circle yes or no.
a. Subtract 5 from both sides.
Yes No
b. Divide both sides by $x$.
Yes No

6. Are either of these an effective first step toward solving for $y$ in the equation $2 a=\frac{1}{8}$ ? Circle yes or no.
a. Subtract $a$ from both sides
Yes No
b. $\quad$ Divide both sides by $\frac{1}{8}$
Yes No 


\section{Algebra Equation Creation Task:}

Now we want you to create algebraic equations. In algebra, variables are used to represent unknown quantities. These variables are represented by letters like $x, y, z$. Algebraic equations can involve both variables and specific numbers. Different arithmetic operations like addition, subtraction, multiplication, and division can be used in the equations.

$$
\begin{array}{ll}
\text { For example: } & \text { addition: } \quad x+8=y \\
& \text { subtraction: } x-8=y \\
& \text { multiplication: } x * 8=y \\
& \text { division: } \quad x / 8=y
\end{array}
$$

You can construct algebraic equations to represent different relations. Consider the following addition algebra problem:

There are 5 more books than magazines. Construct an addition equation to represent this statement.

To construct the equation:

You do not know the number of books or magazines so these can be represented by variables. Let's use $\boldsymbol{B}$ for books and $\boldsymbol{M}$ for magazines.

We know that there are more books than magazines and the relation between them can be expressed with addition as indicated in the question.

To find the number of books, $b$, we need to add 5 to the number of magazines, $m$ since there are 5 more books than magazines.

We can set up the equation like this:

$$
M+5=B
$$

Now try the following problems on your own. Please show your work on the page.

1. Construct a subtraction equation to represent the following statement:

There are 2 more boys than girls.

Use $B$ to represent the number of boys and $\mathbf{G}$ to represent the number of girls

Answer: 
2) Construct a division equation to represent the following statement:

In a certain classroom, there are 4 times as many students as teachers.

Use $\mathbf{S}$ to represent the number of students and $\mathbf{T}$ to represent the number of teachers

Answer: /

3) Construct a division equation to represent the following statement:

A grocery store has 5 times as many apples as baskets

Use $\mathrm{A}$ to represent the number of apples and $\mathrm{B}$ to represent the number of baskets.

Answer: /

4) Construct a multiplication equation to represent the following statement: The florist has 9 times as many flowers as vases.

Use $\mathrm{F}$ to represent the number of flowers and $\mathrm{V}$ to represent the number of vases.

Answer: ${ }^{*}+$

5) Construct a multiplication equation to represent the following statement: There are 7 times as many cookies as jars.

Use $\mathbf{C}$ to represent the number of cookies and $\mathbf{J}$ to represent the number of jars.

Answer: ${ }^{*}+$ 


\section{Highlights}

- Relational fraction knowledge has been linked to algebra performance

- We tested a causal connection between relational fraction knowledge and creating algebra equations during an algebraic modeling task with middle school pre-algebra students and college level adults

- For both age groups, students performance on the subsequent algebra task was higher for students who first performed a relational fractions task compared to a control task

- There was no difference in performance on a similar word problem task

- These findings suggest that 1) relational fractions knowledge enhances algebraic reasoning and 2) these are independent of word problem solving which relies more heavily on procedural knowledge 